\title{
How can skin conductance responses increase over trials while skin resistance responses decrease?
}

\author{
MANFRED VELDEN \\ University of Osnabrück, Osnabrück, West Germany \\ and \\ GERHARD VOSSEL \\ University of Mainz, Mainz, West Germany
}

\begin{abstract}
It is shown that the opposite trends over trials for conductance and resistance response magnitudes that have been found in recent experiments can be explained entirely by the mathematical relationship between conductance and resistance. Two conditions have to be satisfied for such opposite trends to occur: (1) a substantial increase in conductance level over the course of an experiment, and (2) a certain increase in conductance response magnitudes. The opposition in trends, being due solely to the mathematical relationship between conductance and resistance, should not be eliminated by some "correction" procedure. Instead, conductance should be taken as the appropriate variable. Also, no correction should be made for the correlation between conductance level and conductance response magnitude, since it reflects psychological processes rather than a biological level dependency.
\end{abstract}

In a study published recently (Boucsein, Baltissen, \& Euler, 1984), as well as in experiments conducted in our own laboratory, parts of which will be presented below for illustrative purposes, a rather paradoxical effect was observed. With high stimulus intensities, repeated stimulation resulted in an increase in skin conductance responses (SCRs) over trials while, at the same time, the corresponding skin-resistance responses (SRRs) decreased. Since conductance and resistance are related nonlinearly, the pattern of results for electrodermal activity will always differ somewhat, depending on whether it is resistance or conductance that is being taken as the appropriate manifest variable to represent some latent (psychological) variable. With results differing in a qualitative way, as in the experiments mentioned above (measurement of conductance suggests sensitization over trials, whereas resistance indicates habituation), it is well worth asking what the reason for this difference is. This reasoning will have a strong bearing on how to proceed with the data, for example whether or not to apply some correction procedure.

In trying to explain the difference, Boucsein et al. (1984) referred to the correlations they found in their data between level (initial value) and response magnitude. With few exceptions, they found positive level-response correlations for both skin conductance and skin resistance, which they interpreted as a level-dependency of the

Requests for reprints should be sent to $\mathrm{M}$. Velden. University of Osnabrück. Department of Psychology. P.O. Box 4469. D-4500 Osnabrück. West Germany. responses. They explained the opposing trends over trials for resistance and conductance responses in the case of high stimulus intensities by making the assumption that, for these stimulus intensities, an increase in skin conductance level (and, accordingly, a decrease in skin resistance level) occurred over trials. In this case, the positive correlations would mean a tendency for the conductance responses to increase over trials and a tendency for the resistance responses to decrease. The authors interpreted the level-response correlations in terms of a leveldependency, and consequently proposed a correction procedure that would eliminate the opposition found in the trends of the SCRs and the SRRs.

But how can there be positive level-response correlations for both conductance and resistance, with both conductance and resistance responses reflecting the same psychological response? Even though there is a nonlinear relationship between resistance and conductance, larger psychological responses should yield both larger conductance and resistance responses and smaller psychological responses should yield both smaller resistance and conductance responses.

If there is a tendency for larger amplitude psychological responses (and thus greater conductance responses) to be obtained with higher conductance levels, for example, due to increased arousal, then we would expect a positive level-response correlation when measuring conductance, as was found by Boucsein et al. Assuming also that greater resistance responses reflect greater psychological responses, there should, however, be a negative level-response correlation when measuring resistance. 
Greater psychological responses with higher conductance level mean greater psychological responses with lower resistance level, simply because resistance and conductance are being related reciprocally. The reciprocity between resistance and conductance usually does not mean a reciprocity between the resistance- and conductanceresponse amplitudes. But how can such a reciprocity between amplitudes evolve and cause a positive level-response correlation for conductance and resistance? The reason is a purely mathematical one, one that has nothing to do with biological level dependency of electrodermal responses. Methodological implications will be addressed below.

Figure 1 depicts the well-known relationship between conductance and resistance. The $\Delta \mathrm{C}$ segments on the abscissa represent three conductance-response amplitudes; the $\Delta \mathrm{R}$ segments on the ordinate represent the corresponding resistance-response amplitudes, the arrows indicating the direction of change. It can be seen that the conductance amplitudes become increasingly larger from $\Delta \mathrm{C}_{1}$ to $\Delta \mathrm{C}_{3}$ while the corresponding resistance amplitudes become increasingly smaller. For the sake of graphical simplicity, the responses were chosen to be adjacent. It can easily be imagined that the same effect (reciprocity of resistance- and conductance-response amplitudes) would result if every next response started at a level above the upper level of the preceding response. The effect may also occur, to a certain limit, if the $\Delta \mathrm{Cs}$ (and the corresponding $\Delta R s$ ) overlap to some extent. It cannot occur, of course, if all responses start at the same level. In this case, an increase in conductance-response amplitudes would always be accompanied by an increase in the corresponding resistance-response amplitudes. It should be noted that the reciprocal relationship between $\mathrm{R}$ and $\mathrm{C}$ must not imply reciprocity of resistance and conductance response magnitudes, even if there are substantial differences in level. If conductance response amplitudes were to decrease with increasing conductance level, the cor-

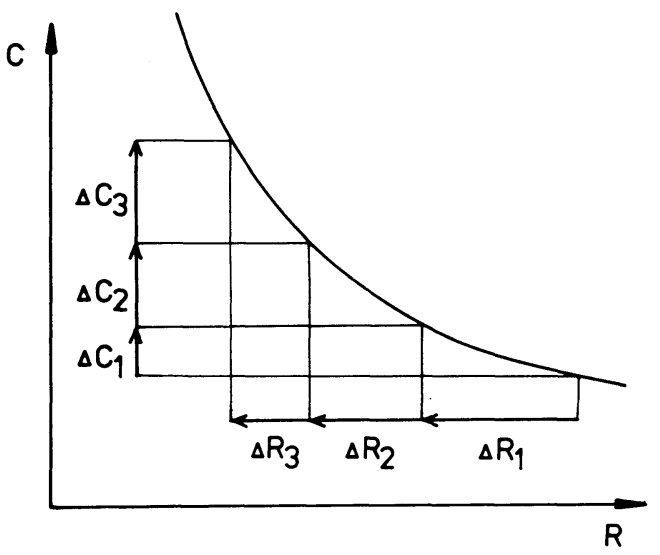

Figure 1. Possible increase in conductance responses to three successive stimulations with a corresponding decrease in resistance responses. responding resistance responses would also decrease, although at a faster pace.

It need hardly be pointed out that the above reasoning implies that an increase in conductance level over trials can mean that a decrease, over trials, in resistance response amplitudes corresponds to an increase in conductance response amplitudes. Whether or not this effect occurs depends, of course, on the amount of change in level over trials. A small or zero level change will result in similar, rather than opposing, trends for resistance- and conductance-response amplitudes, as is usually found (and was also found in the study mentioned above for low stimulus intensities).

In the study by Boucsein et al., no data on level were presented, so it is not possible to determine whether or not the opposing trends observed for resistance and conductance responses were actually due to a substantial shift in level over the whole course of the experiment. Empirical researchers usually tend not to fully accept a deductive proof unless there are empirical data that behave according to that proof. We, therefore, present some illustrative data from two experiments performed in the second author's laboratory. Since the experiments were not designed for this illustrative purpose, only the relevant data will be presented. Skin conductance data covering a broad range of level and response values were selected from a standard habituation experiment (cf. Vossel \& Rossmann, 1985) and from a stress experiment in which subjects had to perform a complex reaction time task in the presence of discrete noise events (cf. Brunner, 1986).

\section{METHOD}

\section{Subjects}

The subjects in the habituation experiment were 36 male students aged 19-27 years. The subjects in the stress experiment were 19 male students, aged 19-36 years.

\section{Apparatus}

Skin conductance measurement. A Schwarzer polygraph (Varioskript V 822) monitored subjects' skin conductance responses (SCRs), skin conductance levels (SCLs), and presentation of stimuli. Beckman $\mathrm{Ag}-\mathrm{AgCl}$ electrodes (surface area $=.64 \mathrm{~cm}^{2}$ ) were placed on the thenar and hypothenar surfaces of the palm of the subject's nondominant hand using Beckman stomaseal electrode collars and Beckman electrode paste as a conducting medium. Skin conductance was detected by a constant-voltage $(.5 \mathrm{~V})$ skin-conductance coupler (Lykken \& Venables, 1971).

Habituation experiment. The stimulus series of the habituation experiment consisted of $2-\mathrm{sec}, 1000-\mathrm{Hz}$ pure sine tones with a randomized interstimulus interval averaging $22.5 \mathrm{sec}$ (range = $15-30 \mathrm{sec}$ ). Tone intensity was $70 \mathrm{~dB}$ (re: $.0002 \mathrm{dyne} / \mathrm{cm}^{2}$ ) as meaured by a sound-level meter (Brüel \& Kjaer Type 2203) at the headrest of the subject's chair. Twenty tone stimuli were presented. They were prerecorded on tape and delivered by a tape recorder (Revox A 77) through a speaker placed against the wall in front of the subject at a distance of approximately $150 \mathrm{~cm}$.

Stress experiment. In the stress experiment, the subject performed a computerized reaction time task. A stimulus board (ZAKDTG) containing 20 colored lights (four each of blue, yellow, red, 
green, and white) was employed. The subject responded to each light by pressing a key with the corresponding color using his dominant hand. Sixty lights were presented per minute.

During performance of the reaction time task, 20 noise bursts (composed of a mixture of white noise and different machine noises) were presented. Noise intensity was $103 \mathrm{~dB}\left(\mathrm{re}: .0002 \mathrm{dyne} / \mathrm{cm}^{2}\right)$, mean noise duration was $10 \mathrm{sec}$ (range $=5-15 \mathrm{sec}$ ), and the interstimulus interval varied between 30 and $90 \mathrm{sec}$ (mean $=60 \mathrm{sec}$ ). Modes of noise delivery and intensity measurement were the same as in the habituation experiment.

\section{Procedure}

The subjects were requested to wash their hands prior to connection of the electrodes. Both experiments took place in a soundproof chamber. Room temperature was between $19^{\circ}$ and $22^{\circ} \mathrm{C}$. All recording and programming equipment was located outside the chamber.

In the habituation experiment, a rest period of $5 \mathrm{~min}$ was followed by 20 presentations of the tone stimuli without further warning. The subject was seated in a semireclining chair and was requested to sit quietly during the whole period.

In the stress experiment, a rest period of $2 \mathrm{~min}$ was followed by the instructions for the reaction time task. The subject was instructed to respond to each light as quickly and accurately as possible. He was further informed that a loud noise would occur from time to time. The main task started after the instruction and a 1-min training phase. It lasted for $24 \mathrm{~min}$ and $20 \mathrm{sec}$, during which the 20 noise bursts were delivered.

\section{Scoring}

SCRs greater than $0.02 \mu$ mhos that occurred between 1 and $5 \mathrm{sec}$ following tone or noise onset were considered as stimulus-evoked responses. They were measured as change in conductance. SCLs were sampled at 20 -sec intervals preceding each tone or noise stimulation. To compare the trends for SCRs and SRRs over trials, the SCRs were transformed into SRRs. It should be noted that the precision of modern skin conductance measurement makes it unneces-

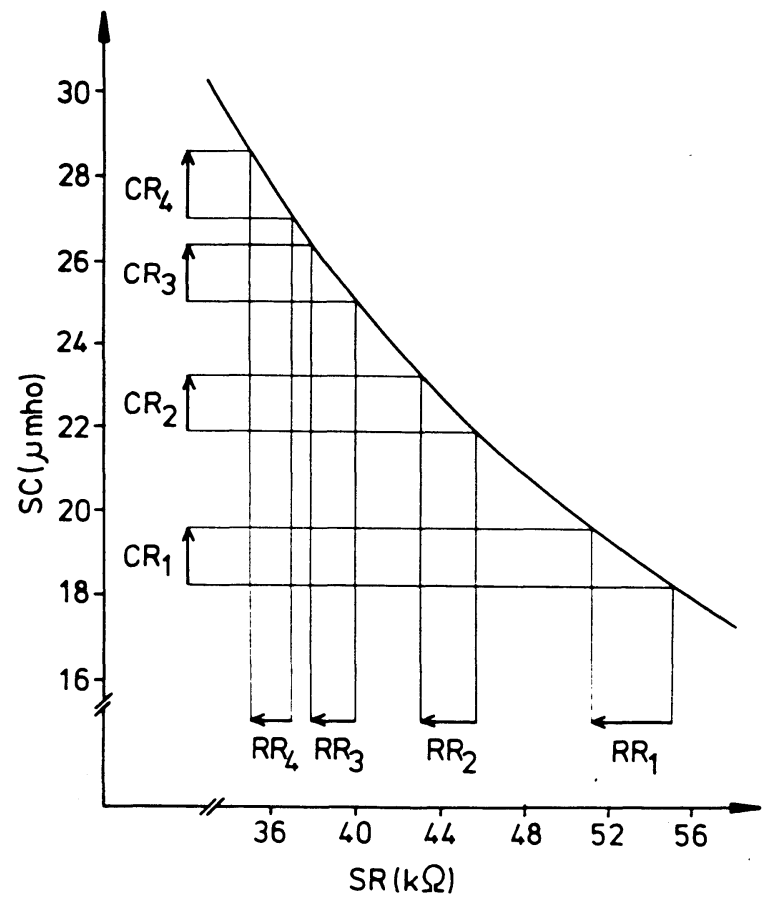

Figure 2. Mean conductance levels and responses of four successive trial blocks with corresponding resistance levels and responses (stress experiment).

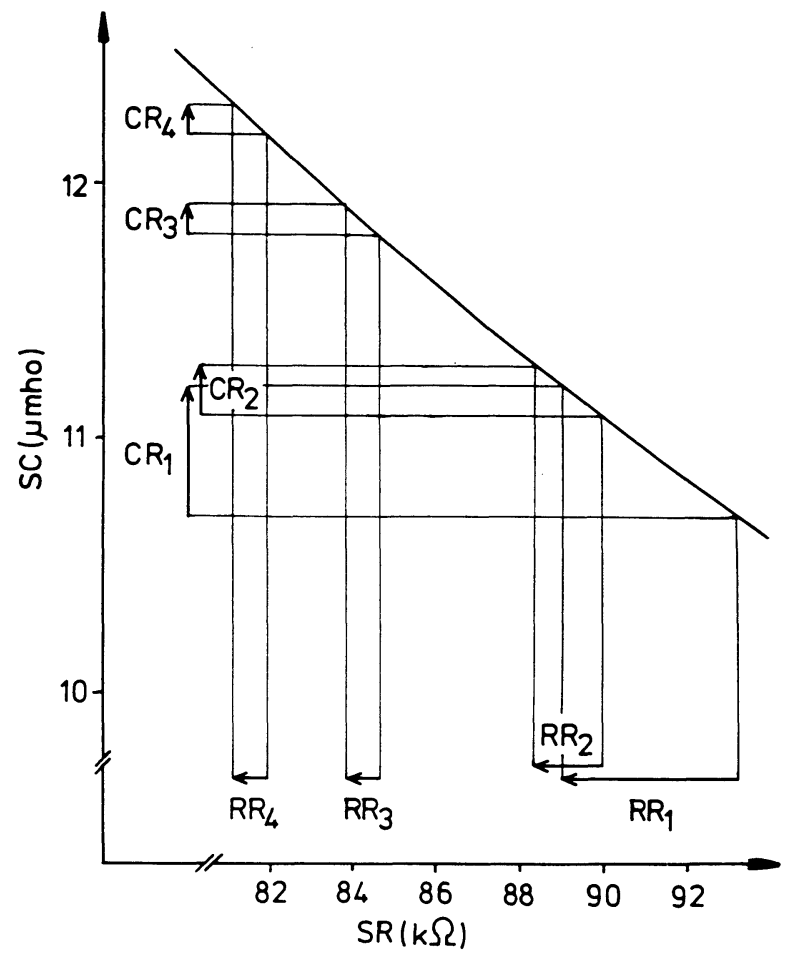

Figure 3. Mean conductance levels and responses of four successive trial blocks with corresponding resistance levels and responses (habituation experiment).

sary to measure both resistance and conductance independently. Boucsein et al. (1984), who did so, actually found no difference in their results when they measured resistance and transformed to conductance and when they measured conductance and transformed to resistance.

\section{RESULTS AND DISCUSSION}

Figure 2 shows the mean conductance-response magnitudes and their corresponding resistance-response magnitudes from the stress experiment, with the total number of trials condensed to four blocks. The responses start at the mean initial level of each trial block. There is a numerically small, but consistent, increase in the size of the conductance responses over trial blocks, accompanied by an obvious decrease in the size of the resistance responses. The rather small decrease in resistance responses from the third to the fourth trial block is due to the rather strong increase in the sizes of the conductance responses. Had the conductance responses increased still more from the third to the fourth trial block, there might have been an increase in the corresponding resistance responses.

Figure 3 shows the data of the habituation experiment with the same kind of data reduction as in Figure 2. In this case, a decrease can be seen in the size of the conductance responses over trial blocks, as can, correspondingly, an even somewhat more pronounced decrease in the size of the resistance responses, due to the nonlinearity of the relation between $C$ and $R$. Note that the shift 
in level under this experimental condition is numerically very small relative to the one for the stress experiment. The effect of the small size of the segment of the curve representing the relationship between $\mathrm{R}$ and $\mathrm{C}$ that is depicted in Figure 3 is that it appears to be nearly linear. This means that, had there been a pronounced increase in the size of the conductance responses within the range of levels shown in Figure 3, there could well have been an increase in the size of the resistance responses. This shows that, even under the condition of increasing levels of conductance and increasing conductance response amplitudes, the result need not be opposing trends for conductance and resistance responses. It all depends on both the size of the level shift and the amount of change in response amplitudes over the course of the experiment.

For an overview, the results of the two experiments are taken together and presented in a more common form in Figure 4, with the conductance data being depicted on the left and the resistance data on the right. The qualitative difference between the trends for the resistance and conductance responses in the stress experiment as opposed to the concordance found in the habituation experiment can be seen very clearly.

These methodological considerations have strong implications for the question of "correction" procedures. As mentioned above, Boucsein et al. proposed a correction procedure that eliminated the difference between the patterns of results found for resistance and conductance.

First it should be kept in mind that, in a psychophysiological laboratory, skin resistance and conductance are measured in such a way as to reflect the same biological (electrodermal activity) and psychological (e.g., attention, arousal, etc.) entity, even though in different forms,

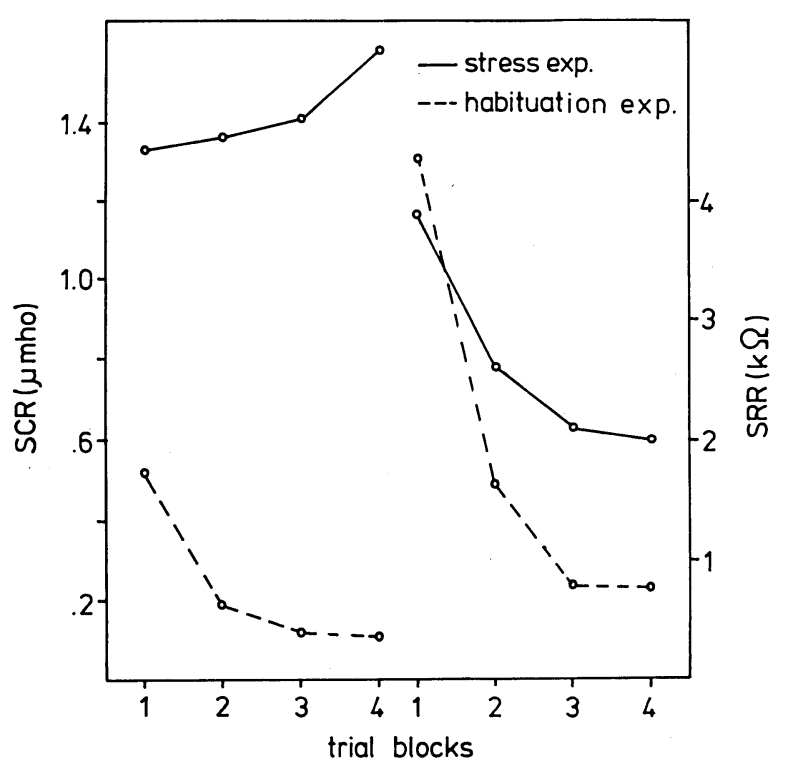

Figure 4. Conductance and resistance response amplitudes over trial blocks for stress and habituation experiments. reflecting their nonlinear relationship to each other. They are not correlated, but they are related mathematically. This means that any difference between resistance and conductance data, like the opposing trends over trials found, for high stimulus intensities, both by Boucsein et al. and in one of our experiments, is exclusively due to the mathematical relationship between resistance and conductance. Under certain circumstances (sufficient shift in level and some increase in conductance response amplitudes over trials), opposing trends must occur for resistance and conductance response magnitudes. It clearly makes no sense to apply a correction procedure in order to eliminate differences in trends that must occur for mathematical reasons. It must be asked, instead, which of the two physical variables is the appropriate one-that is, the one that is possibly related linearly to the latent (psychological) variable. Actually, conductance has generally been accepted as the appropriate variable in the psychophysiological laboratory, and the reasons that have been put forward in the literature for this will not be repeated here. It should be mentioned, however, that the data presented above also favor conductance as the appropriate variable. With the high stimulus intensities in the stress experiment, subjects probably became increasingly aroused over the course of the experiment (as reflected in both the increase in conductance level and the decrease in resistance level), which means that they became increasingly responsive to the stimuli, as reflected in the conductance-response, but not the resistanceresponse, amplitudes.

Note that this implies that there is also no biological level dependency for which to make some kind of correction. If the subjects became increasingly aroused over trials and therefore reacted with increasing responses, which is most plausible, then there must have been some kind of psychological level dependency which should not be eliminated by some correction procedure. As argued by Lykken and Venables (1971), care should be taken not to use correction procedures that might eliminate valuable psychological information. A biological level dependency in terms of a ceiling effect (which should be corrected for) would require that the conductance responses decrease with increasing conductance level.

The following is a summary of the essential points: Opposing trends, over trials, in the magnitudes of conductance and resistance responses may occur under certain circumstances (substantial increase of skin conductance level and some increase in skin conductance response magnitude over trials) for purely mathematical reasons. No "correction" should therefore be made to eliminate the difference between the trends; instead, conductance should simply be taken as the appropriate variable. Finally, the correlation between conductance level and conductance response magnitude for high stimulus intensities is not due to a biological level dependency, but reflects psychological processes; this also argues against the use of data "correction" procedures. 


\section{REFERENCES}

Boucsein, W., Baltissen, R., \& Euler, M. (1984). Dependence of skin conductance reactions and skin resistance reactions upon'previous level. Psychophysiology, 21, 212-218.

BRUNNER, R. (1986). Nacheffekte von Stress: Eine Überprüfung der "information load" Hypothese von Cohen. Unpublished master's thesis, University of Mainz.

LyKKen, D. T., \& Venables, P. H. (1971). Direct measurement of skin conductance: A proposal for standardization. Psychophysiology, 8, 656-672.

Vossel, G., \& Rossmann, R. (1985). Stabilität und Konsistenz der EDAHabituationsgeschwindigkeit. Zeitschrift fü Differentielle und Diagnostische Psychologie, 6, 10-18.

(Manuscript received August 15, 1985; accepted for publication December 5, 1985.) 